\title{
ACMG position statement on prenatal/preconception expanded carrier screening
}

\author{
Wayne W. Grody, MD, PhD 1,2, Barry H. Thompson, MD, MS 3 , Anthony R. Gregg, MD4, \\ Lora H. Bean, $\mathrm{PhD}^{5}$, Kristin G. Monaghan, $\mathrm{PhD}^{6}$, Adele Schneider, $\mathrm{MD}^{7}$ and Roger V. Lebo, $\mathrm{PhD}^{8}$
}

\begin{abstract}
Disclaimer: ACMG position statements are developed primarily as educational resources for medical geneticists to help them provide quality clinical laboratory genetic services. Adherence to this statement is voluntary and does not necessarily ensure a successful medical outcome. This position statement should not be considered inclusive of all proper procedures and tests or exclusive of other procedures and tests that are reasonably directed to obtaining the same results. In determining the propriety of any specific procedure or test, the clinical laboratory geneticist should apply his or her own professional judgment to the specific circumstances presented by the individual patient or specimen. Clinical laboratory geneticists are encouraged to document in the patient's record the rationale for the use of a particular procedure or test, whether or not it is in conformance with this position statement. They also are advised to take notice of the date any particular position statement was adopted and to consider other relevant medical and scientific information that becomes available after that date. It also would be prudent to consider whether intellectual property interests may restrict the performance of certain tests and other procedures.
\end{abstract}

For years, clinicians have offered gene-by-gene carrier screening to patients and couples considering future pregnancy or those with an ongoing pregnancy early in gestation. Examples include ethnicspecific screening offered to Ashkenazi Jewish patients and panethnic screening for cystic fibrosis and spinal muscular atrophy. Nextgeneration sequencing methods now available permit screening for many more disorders with high fidelity, quick turnaround time, and lower costs. However, instituting these technologies carries with it perils that must be addressed. The basis for the selection of disorders on expanded carrier screening panels should be disclosed. The information provided about disorders with mild phenotypes, variable expression, low penetrance, and/or characterized by an adult onset should be complete and transparent, allowing patients to opt out of receiving these test results. Patients also must be made aware of the concept of residual risk following negative test results. Laboratories have a duty to participate in and facilitate this information transfer.

Genet Med 2013:15(6):482-483

Key Words: expanded carrier screening; prenatal genetic screening

preconception screening and diagnosis. Now it is possible, using new technologies, to screen for mutations in many genes for approximately the same cost as previously required to detect mutations in a single gene or a relatively small number of population-specific mutations in several genes. Commercial laboratories have begun to offer such expanded carrier screening panels to physicians and the public, but there has been no professional guidance on which disease genes and mutations to include.

The proper selection of appropriate disease-causing targets for general population-based carrier screening (i.e., absence of a family history of the disorder) should be developed using clear criteria, rather than simply including as many disorders as possible. For a particular disorder to be included in carrier screening, the following criteria should be met:

${ }^{1}$ Division of Medical Genetics, UCLA School of Medicine, Los Angeles, California, USA; ${ }^{2}$ Division of Molecular Diagnostics, UCLA School of Medicine, Los Angeles, California, USA; ${ }^{3}$ American College of Medical Genetics and Genomics, Bethesda, Maryland, USA; ${ }^{4}$ Department of Obstetrics and Gynecology, University of Florida College of Medicine, Gainesville, Florida, USA; ${ }^{5}$ Emory Genetics Laboratory, Division of Medical Genetics, Department of Human Genetics, Emory University, Atlanta, Georgia, USA; ${ }^{6}$ Department of Medical Genetics, Henry Ford Hospital, Detroit, Michigan, USA; ${ }^{7}$ Genetics Division, Department of Pediatrics, Albert Einstein Medical Center, Philadelphia, Pennsylvania, USA; ${ }^{8}$ Department of Pathology and Laboratory Medicine, Akron Children’s Hospital, Akron, Ohio, USA. Correspondence: Wayne W. Grody (wgrody@mednet.ucla.edu) 
1. Disorders should be of a nature that most at-risk patients and their partners identified in the screening program would consider having a prenatal diagnosis to facilitate making decisions surrounding reproduction.

- The inclusion of disorders characterized by variable expressivity or incomplete penetrance and those known to be associated with a mild phenotype should be optional and made transparent when using these technologies for screening. This recommendation is guided by the ethical principle of nonmaleficence.

2. When adult-onset disorders (disorders that could affect the offspring of the individual undergoing carrier screening once the offspring reaches adult life) are included in screening panels, patients must provide consent to screening for these conditions, especially when there maybe implications for the health of the individual being screened or other family members.

- This recommendation follows the ethical principles of autonomy and nonmaleficence.

3. For each disorder, the causative gene(s), mutations, and mutation frequencies should be known in the population being tested, so that meaningful residual risk in individuals who test negative can be assessed.

- Laboratories should specify in their marketing literature and test results how residual risk was calculated using panethnic population data or a specific race/ethnic group.

- The calculation of residual risk requires knowledge of two factors: one is the carrier frequency within a population, the other is the proportion of disease-causing alleles detected using the specific testing platform. Laboratories using multiplex platforms often have limited knowledge of one or both factors. Laboratories offering expanded carrier screening should keep data prospectively and regularly report findings that allow computation of residual risk estimates for all disorders being offered. When data are inadequate, patient materials must stress that negative results should not be overinterpreted.

4. There must be validated clinical association between the mutation(s) detected and the severity of the disorder.

- Patient and provider materials must include specific citations that support inclusion of the mutations for which screening is being performed.
5. Compliance with the American College of Medical Genetics and Genomics Standards and Guidelines for Clinical Genetics Laboratories, including quality control and proficiency testing.

- Quality control should include the entire test process, including preanalytical, analytical, and postanalytical phases. Test performance characteristics should be available to patients and providers accessing testing.

A highly multiplexed approach will require a more generic consent process than is typically used for single-disease screening because it may not be practical for a clinician to discuss each disease included in a multidisease carrier screening panel. An appropriately tailored informational pamphlet or Web site, containing a brief description of each disorder included in a test panel, should be available to patients undergoing or considering an expanded prenatal/preconception carrier screening panel. Genetic counseling before testing should be available to those who desire this, and posttest genetic counseling for those with positive screening results is recommended. A recent categorization of risk denotes carrier screening as moderate risk. ${ }^{1}$ Posttest counseling should include disclosure of the mutation(s) detected, description of the clinical nature (including the natural history and management) of the disorder in question, facilitation of testing of the reproductive partner, calculation of the revised fetal risk when the partner is not available for testing or declines testing, identification of other family members at risk for the disorder, and discussion of options for fetal testing and reproductive decision-making.

\section{DISCLOSURE}

B.H.T., A.R.G., and A.S. declare no conflict of interest. W.W.G., L.H.B., and K.G.M. direct molecular genetics laboratories that perform carrier screening. R.V.L. directs a laboratory that performs confirmatory testing and carrier screening for cystic fibrosis.

\section{REFERENCE}

1. Monaghan KG, Benkendorf J, Cherry AM, et al. Risk categorization for oversight of laboratory-developed tests for inherited conditions. Genet Med 2013;15: 314-315. 